\title{
Improved Surgical Outcomes of Laparoscopic Gastrectomy Compared with Conventional Open Gastrectomy for Gastric Adenocarcinoma in Obese Patients
}

\author{
Sung II Choi, M.D., Ph.D. \\ Department of Surgery, Kyung Hee University Hospital at Gangdong, Seoul, Korea
}

Corresponding author

Sung Il Choi

Department of Surgery, Kyung Hee University Hospital at Gangdong, Kyung Hee Univesity College of Medicine, 892 Dongnam-ro, Gangdong-gu, Seoul 05278, Korea

Tel: +82-2-440-6136, Fax: +82-2-440-6073, E-mail: drchoi@khu.ac.kr

Laparoscopic abdominal surgery including gastrectomy in obese patients is challenging. Obesity has been increasing and more and more patients we encounter in the surgical clinic are obese as well. ${ }^{1}$

Although the incidence of gastric cancer has been on the decline, it is still one of most common cancers in Asian countries. ${ }^{2}$ Minimally invasive surgery for gastric cancer is being practiced more often with the development of new instruments and improvement in devices. The short and long term results regarding the safety and efficacy of oncological outcomes of laparoscopic gastrectomy in early gastric cancer have already been established and are considered unequivocal. ${ }^{3,4}$ We are expecting such results in advanced gastric cancer.

Obese patients with gastric cancer have shown poor surgical outcomes in open and laparoscopic surgery compared with normal weight patients. A longer operation time, more intraoperative blood loss, and less lymph node retrievals were reported and more evident in cases of open surgery. ${ }^{5}$

A high level of surgical technique is required in every process in obese gastric cancer patients, from inserting the trocars, performing gastrectomy with adequate lymph node dissection and completing reconstruction. Excessive fat makes anatomical dissection difficult during omentectomy, lymph node dissection around major vessels and in conducting a tension-free reconstruction.

Short term outcomes of laparoscopic surgery in obese patients such as operation time, open conversion rate, postoperative complications and mortality are concerning issues for the surgeon. Furthermore, long term results including cancer recurrence and incisional hernia are of interest.

In this article, the author divided the patients into two groups according to their BMI. However, the extent of visceral obesity could be of more importance in abdominal surgery because BMI may not accurately reflect the amount of intraabdominal fat, which makes laparoscopic procedures more difficult. ${ }^{6}$ The results of this study might differ if data were reanalyzed according to visceral fat.

The patients in the open gastrectomy group had slightly larger tumors and had received more aggressive lymph node dissection than in the laparoscopic group even though all patients were diagnosed with early gastric cancer. This is one of the reasons why the laparoscopic group had more favorable results. Surprisingly, the operation time was shorter in the laparoscopic group, which was probably because of the well trained and highly qualified participating operators. This article helps readers consider the advantages of laparoscopic 
gastrectomy over open gastrectomy performed by a skillful surgeon in obese patients.

Recently, totally laparoscopic gastrectomy (TLG) has been thought to have more advantages than laparoscopic-assisted gastrectomy (LAG) with respect to pain, scar and recovery time to daily life ${ }^{7}$ because TLG with intracorporeal anastomosis requires a smaller incision for specimen extraction alone, especially in obese patients. A similar study comparing LAG and TLG in obese gastric cancer patients would yield interesting results.

\section{REFERENCES}

1) Farzadfar F, Finucane MM, Danaei G, et al. National, regional, and global trends in serum total cholesterol since 1980: systematic analysis of health examination surveys and epidemiological studies with 321 country-years and 3.0 million participants. Lancet 2011;377:578-586.

2) Ferlay J, Soerjomataram I, Dikshit R, et al. Cancer incidence and mortality worldwide: sources, methods and major patterns in
GLOBOCAN 2012. Int J Cancer 2015;136:E359-386.

3) Kim HH, Hyung WJ, Cho GS, et al. Morbidity and mortality of laparoscopic gastrectomy versus open gastrectomy for gastric cancer: an interim report--a phase III multicenter, prospective, randomized Trial (KLASS Trial). Ann Surg 2010;251:417-420.

4) Kim HH, Han SU, Kim MC, et al. Prospective randomized controlled trial (phase III) to comparing laparoscopic distal gastrectomy with open distal gastrectomy for gastric adenocarcinoma (KLASS 01). J Korean Surg Soc 2013;84:123-130.

5) Li L, Li X, Chu S, et al. Does overweight affect outcomes in patients undergoing gastrectomy for cancer? A meta-analysis of 25 cohort studies. Jpn J Clin Oncol 2014;44:408-415.

6) Shin HJ, Son SY, Cui LH, et al. Is There any Role of Visceral Fat Area for Predicting Difficulty of Laparoscopic Gastrectomy for Gastric Cancer? J Gastric Cancer 2015;15:151-158.

7) Chen K, Pan Y, Zhai ST, et al. Laparoscopic gastrectomy in obese gastric cancer patients: a comparative study with non-obese patients and evaluation of difference in laparoscopic methods. BMC Gastroenterol 2017;17:78. 